Hydrology and Earth System Sciences, 8(1), 2-7 (2004) (C) EGU

\title{
Direct measurement of the soil water retention curve using X-ray absorption
}

\author{
A. Bayer, H.-J. Vogel and K. Roth \\ Institute of Environmental Physics, INF 229, University of Heidelberg, D-69120 Heidelberg, Germany \\ E-mail for corresponding author: andreas.bayer@iup.uni-heidelberg.de
}

\begin{abstract}
X-ray absorption measurements have been explored as a fast experimental approach to determine soil hydraulic properties and to study rapid dynamic processes. As examples, the pressure-saturation relation $\theta(\psi)$ for a uniform sand column has been considered as has capillary rise in an initially dry sintered glass column. The $\theta(\psi)$-relation is in reasonable agreement with that obtained by inverting a traditional multi-step outflow experiment. Monitoring the initial phase of capillary rise reveals behaviour that deviates qualitatively from the single-phase, localequilibrium regime described by Richards' equation.
\end{abstract}

Keywords: X-ray absorption, soil hydraulic properties, soil water dynamics, Richards' equation

\section{Introduction}

The determination of hydraulic properties of porous media is time-consuming and afflicted with serious uncertainties. Particular difficulties arise near water saturation where multiphase phenomena may be expected to be important. To measure the distribution of water in porous media, X-ray absorption can be useful. Measurements can be made with high spatial and temporal resolution but high spatial resolution demands long exposure times and so reduces temporal resolution. In X-ray computed tomography, a complete scan may take several minutes so that faster dynamic effects cannot be observed. Therefore, single slices through the sample are often preferred for monitoring rapid internal changes (Hopmans et al., 1992; Brown et al., 1987).

$\mathrm{X}$-ray absorption methods, especially in computed tomography, were used to detect the inner structure of soil columns in non-destructive and non-invasive measurements (Wildenschild et al., 2002; Ketcham and Carlson, 2001). Furthermore, the distribution of water can be detected by these methods as it has been shown by Hopmans et al. (1992) for coarse sand and sandy loam; they also calculated hydraulic properties by optimising parameters for hydraulic functions. Using dual-energy X-ray absorption techniques, it is also possible to monitor the water content in non-rigid media (Garnier et al., 1998; Rogasik et al., 1999).Using Xray radiography, Mori et al. (1999) visualised drainage patterns and characterised geometric and hydraulic properties of structured soils.

In the present study, projected data were used through single slices of the sample to determine water content at different heights with temporal resolutions down to $1 \mathrm{~s}$. The objectives were to measure the vertical distribution of water in porous media with a high spatial resolution and to determine hydraulic properties. Data with high temporal resolution were used to visualise dynamic effects during the capillary rise of water into a dry porous medium.

\section{Material and methods}

Absorption measurements were made using a polychromatic medical X-ray tube operated at $141 \mathrm{kV}$ and $5 \mathrm{~mA}$ together with a 12-bit CCD line detector with 1280 pixels of $0.4 \mathrm{~mm}$ side length. The tube and detector were mounted $1.32 \mathrm{~m}$ apart and they could be moved synchronously in a vertical direction. The samples to be scanned were centred $1.15 \mathrm{~m}$ from the tube. Measuring time for a single slice was $1 \mathrm{~s}$.

In the absence of a dual-energy X-ray source, the porous medium had to be rigid such that any change in the 
absorption could be attributed to a change in the water content. To this end, sand columns and cylinders of sintered glass were used.

Two types of experiments were effected: (i) the stationary distribution of water above a water table was measured and from this a parameterisation of $\theta(\psi)$ was estimated (ii) the capillary rise of water during imbibition of a dry column was monitored to test the validity of the Richards' equation for the rapid initial phase.

\section{Data processing}

Measured X-ray intensities of each pixel were corrected for the dark current of the respective detector pixel. Then, the product $\hat{\mu}$ of the effective absorption coefficient $\mu^{*}$ and the length of path of the photons through the column $d$ for every pixel was calculated using:

$$
\hat{\mu}=\mu^{*} d=\ln \left(\frac{I_{0}}{I(d)}\right)
$$

where $I_{0}$ is the initial and $I(d)$ the corrected X-ray intensity of the respective pixel. Beam hardening effects resulting from the use of a polychromatic source were neglected as was the energy dependent absorption coefficient. This is permissible because of the moderate variation of path length in the column and homogeneity of the material the beam passes. Differences that originate from beam hardening in the observed integral values should thus be much smaller than differences caused by changes of water content. In Eqn. (1) the absorption coefficient $\mu^{*}(z)$ at sample height $z$ is the sum of the length-fraction weighted absorption coefficients of the different components, hence

$$
\begin{gathered}
\hat{\mu}(z)=\mu^{*}(z) d=(1-\phi) d \mu_{\text {matrix }}+d_{\text {wall }} \mu_{\text {wall }} \\
+\theta(z) d \mu_{\mathrm{H}_{2} \mathrm{O}}+(\phi-\theta(z)) d \mu_{\text {air }},
\end{gathered}
$$

where $\mu_{\text {matrix }}, \mu_{\text {wall }}, \mu_{\mathrm{H}_{2} \mathrm{O}}$ and $\mu_{\text {air }}$ are the absorption coefficients of matrix, the column wall, water and air, respectively, $d$ and $d_{\text {wall }}$ are the thicknesses of the column and its wall, respectively, $\phi$ is the porosity and $\theta(z)$ is the volumetric water content. Neglecting absorption of air whose absorption coefficient is three orders of magnitude lower than that of the other materials present, the last term in Eqn. (2) disappears and the water content is:

$$
\theta(z)=\frac{\hat{\mu}(z)-\hat{\mu}_{0}}{\hat{\mu}_{s}-\hat{\mu}_{0}} \theta_{s},
$$

where $\hat{\mu}_{0}$ is the value calculated using Eqn.(1) of the dry sample, $\hat{\mu}_{s}$ of the completely saturated sample and $\theta_{s}$ is the saturated water content assumed equal to porosity $\phi$. Note that once the column is measured in dry and saturated state, the absorption coefficients of the individual materials need not be known.

In the stationary state, the water potential of a rigid porous medium is $\psi_{w}=\psi+z$, where $\psi$ is the matrix potential and $z$ represents the gravitational potential with $z$ pointing upwards. Here and in the following, potentials are given in terms of equivalent heights of water columns. Hence, in a stationary state, where $\psi_{w}=$ const, the $z$-axis is also a $\psi$ axis. So the water content versus the matrix potential $\psi$ is directly observable.

Hence, the pressure-saturation relation $\theta(z)=\theta(\psi)$ in the stationary experiments can be fitted by the parameterisation of van Genuchten (1980)

$$
\theta(\psi)=\theta_{r}+\left(\theta_{s}-\theta_{r}\right)\left(1+(\alpha \psi)^{n}\right)^{-1+1 / n}
$$

using the known saturated water content $\theta_{s}$.

\section{Stationary experiments}

The first measurement was made on a sand sample filled homogeneously in a PVC (polyvinyl chloride) column $10 \mathrm{~cm}$ high and with a radius of $8.15 \mathrm{~cm}$. The column was mounted on an PVC-apron that included all the features to control the pressure in the water phase at the lower boundary. The top end of the column was not sealed, but was covered with PVC to reduce the moisture exchange between the surface and the atmosphere to minimise evaporation. This cover does not affect the pressure control at the bottom. The grain-size ranged from $0.63 \mathrm{~mm}$ to $1.25 \mathrm{~mm}$. The format of the experimental setup was a simple X-ray absorption layout (Fig. 1). The detector was oriented horizontally (Fig. 1 setup A). Moving detector and tube synchronously from the bottom to the top of the sample determined the vertical distribution of water inside the column. A complete scan was done in less than five minutes. Scans of the dry and saturated columns were made once to obtain reference values of $\hat{\mu}_{0}$ and $\hat{\mu}_{s}$, respectively (Eqn.(3)). To assure the complete water saturation of the sample, sand was filled continuously into the PVC-column, while the water level was readjusted so that the sand surface was always covered with water to avoid air entrapment during filling. Then, the column was desaturated by application of a fixed potential at the lower boundary of $\psi=-3 \mathrm{~cm}$ for about 20 hours and scanned again. After this, the multi-step outflow experiment described in the next paragraph was performed. Finally, to measure the dry sand column, it was dried in an oven for five days at $50^{\circ} \mathrm{C}$ to prevent damage to the surrounding PVC parts. Throughout the experiment, the weight of the column was monitored until it remained constant. Inspection of the X-ray data showed that bulk density was constant with depth. 


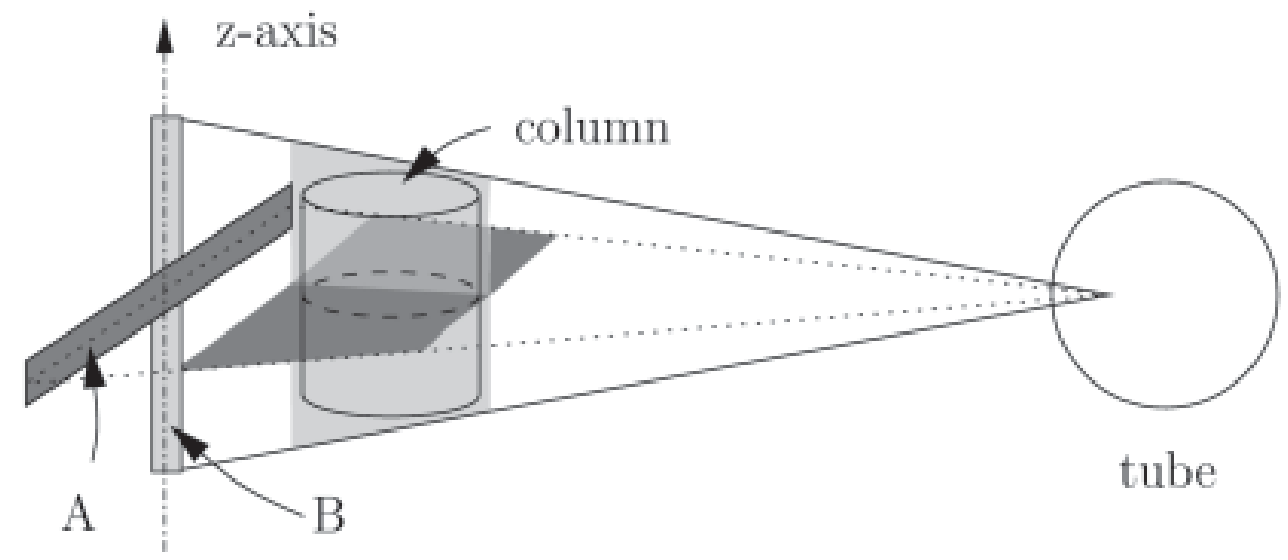

Fig. 1. Side view of the experimental setup with horizontal detector in setup A (dark grey) and and vertical in setup B (bright grey), the X-ray tube and the sample. The shaded areas indicate the slices through the centre of the sample the X-ray beam passed in setup A or B, respectively.

However, the dried column showed a decreased absorption coefficient in the upper $2 \mathrm{~cm}$ of the sample; this is attributed to a higher bulk density produced during the measurements and drying procedure of the sample. Consequently, the data from this interval were not used for fitting. The vertical spatial resolution, $0.4 \mathrm{~mm}$, was determined by the size of the detector pixels. The calculated values for $\hat{\mu}(z)$ Eqn. (1) were averaged over 370 pixels in the horizontal around the centre of the sample to increase the signal to noise ratio on the assumption of spatial uniformity.

The multi-step outflow experiment (van Dam et al., 1994) was run with the same column to estimate the hydraulic parameters. The column was saturated again by raising the water table slowly. A standard setup was used with a porous

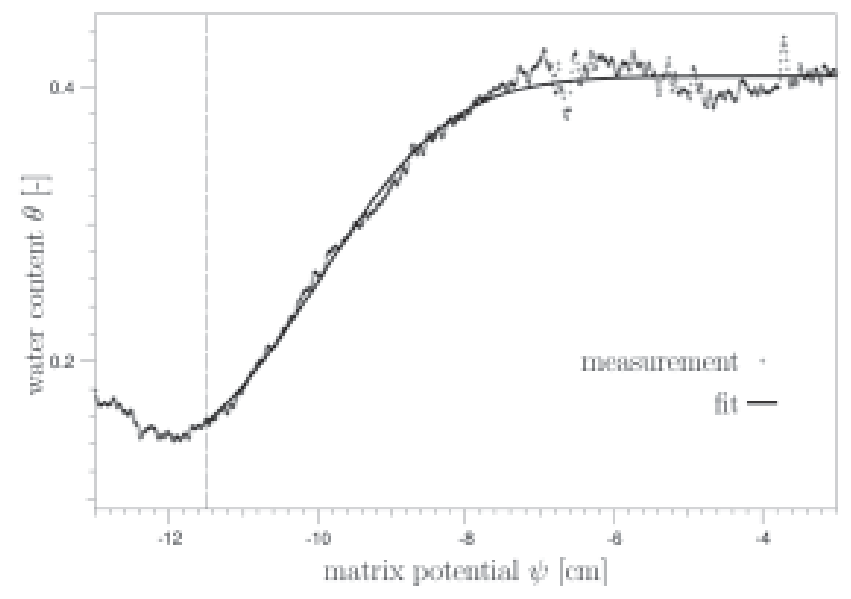

Fig. 3. Multi-step outflow data (cumulative outflow: circles, matrix potential $8 \mathrm{~cm}$ above lower boundary: triangles) during desaturation (pressure at lower boundary: dash-dot) and the model prediction with values determined by $X$-ray absorption (solid line). Additionally the best-fit of the model (dotted line) is shown. The modelled curves for tensiometer data are on top of each other. The bright grey curve is simulated with the parameters of the X-ray absorption but using the same $\alpha$ as in the best-fit model. plate at the bottom of the column. Initially, the material was completely water saturated, i.e. the potential at the lower boundary was $\psi=+10 \mathrm{~cm}$; thereafter the potential at the lower boundary was decreased in ten steps of $1 \mathrm{~cm}$ and one minute duration from $\psi=+10 \mathrm{~cm}$ to $\psi=0 \mathrm{~cm}$ and, then, five steps with $\Delta \psi=-3 \mathrm{~cm}$ and of about one hour duration (Fig. 2). The porosity of the column was determined as $\phi=0.396 \pm 0.002$ from its volume and the mass of the sand used. In a separate experiment, the saturated hydraulic conductivity, measured by the falling-head method (Klute, 1986), was $K_{s}=14 \mathrm{mh}^{-1}$.

\section{Dynamic experiments}

For the measurements at high temporal resolution, setup B (Fig. 1) was used with tube and detector mounted vertically during the experiment. The height of the beam focus of the source was half the height of the sample. This setup allowed the X-ray absorption in a vertical slice through the sample to be monitored with frequencies up to $1 \mathrm{~Hz}$. This increase in temporal resolution was associated with a loss of spatial resolution. With the given geometry, slices through the sample projected the whole sample height onto 270 pixels of the detector. Because of the fan beam geometry, the spatial resolution half way up the column equalled the size of the detector pixel and decreased towards the top and bottom respectively.

This experiment used a column of sintered borosilicate glass $150 \mathrm{~mm}$ in diameter, $100 \mathrm{~mm}$ high with porosity $\phi=0.40$ according to the manufacturer. Since the column was rigid, the PVC tube was put aside and the sintered glass was coated with silicone to prevent unwanted flow of water and air.

Initially, the column was oven-dry. It was then mounted on an apron so that the bottom of the column could be 
connected to a water table and the potential controlled remotely. Recording the transmitted X-ray intensity started immediately after the water phase had been connected to the column. Data were collected in time series at increasing time intervals with individual measurements within a group separated by $1 \mathrm{~s}$. To measure the saturated reference values $\hat{\mu}_{s}$ the column was saturated in an exsiccator at the end of the experiment.

\section{Results and discussion}

\section{STATIONARY EXPERIMENTS}

The pressure-saturation relationship was determined over a range of $\psi=-13, \ldots,-3 \mathrm{~cm}$ (Fig. 3). Optimal values of parameters $n, \alpha$ and $\theta_{r}$ were estimated using the nonlinear least square fit routine implemented in Microcal Origin 6.0 (Microcal, 1997) (Table 1). On the right hand side of Fig. 3, the X-ray signal was disturbed by the apron on which the column was mounted. There, the PVC wall was about five times thicker than that in the upper part of the column. Therefore, the signal to noise ratio was reduced in this range. The part between $\psi=-13, \ldots,-11.5 \mathrm{~cm}$ was affected by changes in the bulk density at the top of the sample and, therefore, these data were not included in the analysis. The scanned interval of $\psi$ can be altered by changing the pressure at the lower boundary; this would allow $\theta(\psi)$ to be scanned for a wider range of potentials, depending on the material.
Table 1. Parameters determined by fitting the van Genuchten parameterisation to the pressure-saturation relation measured by X-ray absorption and from solving the inverse problem for the multi-step outflow data. Uncertainties are calculated using the $\chi^{2}$ statistics as described by Press et al. (1992).

\begin{tabular}{lll}
\hline Parameter & X-ray-abs. & MSO-Fit \\
\hline $\mathrm{n}[-]$ & $10.7 \pm 0.1$ & $11.6 \pm 0.2$ \\
$\alpha[\mathrm{cm}]$ & $9.95 \pm 0.01$ & $9.46 \pm 0.04$ \\
$\theta_{\mathrm{r}}[-]$ & $0.08 \pm 0.01$ & $0.09 \pm 0.01$ \\
\hline
\end{tabular}

The parameters from Table 1 were used with values of porosity determined independently and the saturated hydraulic conductivity to predict the result of the multi-step outflow experiment (Fig. 2).

A second set of van Genuchten parameters is estimated from the multi-step outflow experiment performed with the identical sand column by solving the inverse problem of the Richards' equation. A one-dimensional, fully-implicit, finite difference mixed formulation (Celia et al., 1990) was used with a Levenberg-Marquardt algorithm to optimise the parameters (Zurmüehl, 1994). The best-fit outflow curve and parameters are given in Fig. 2 and Table 1. This second set of parameters leads to a better description of the outflow data because they are fitted directly to the measured outflow data and parameters $\theta_{s}$ and $K_{s}$, having been determined independently, were not fitted to outflow data.

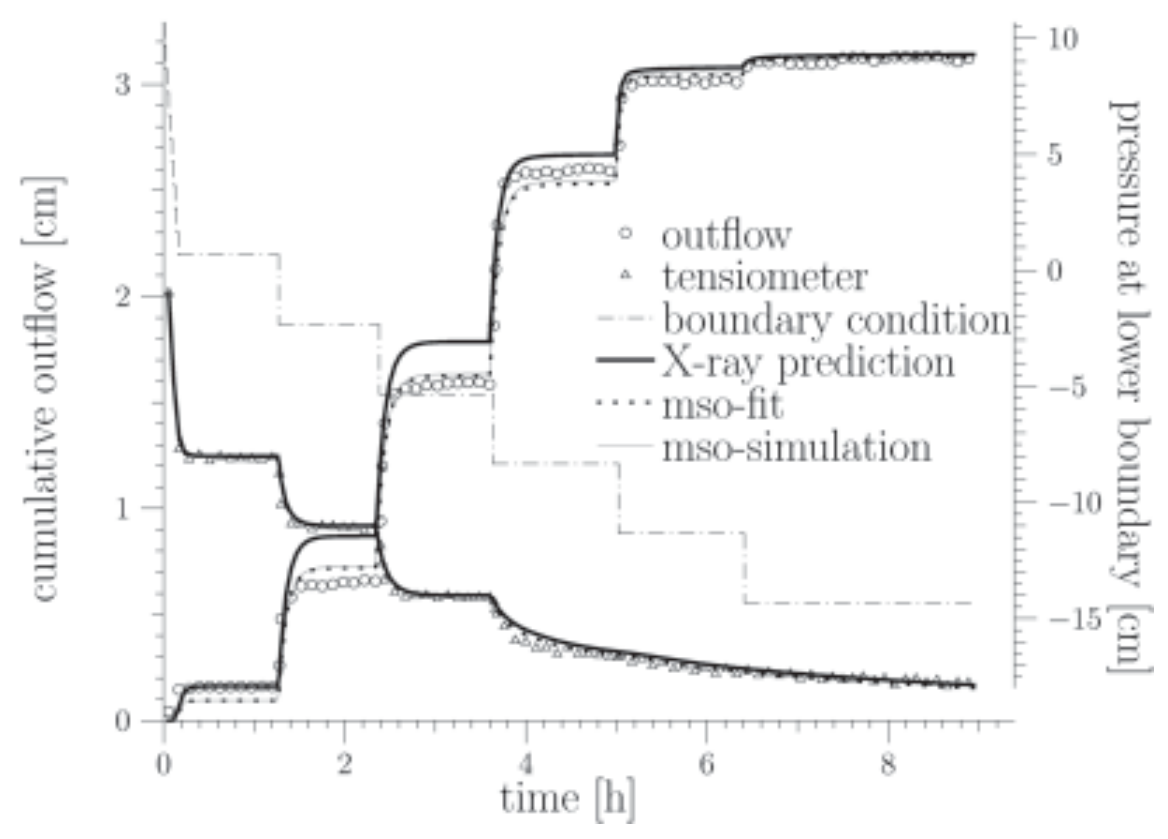

Fig. 2. Pressure-saturation relation of coarse sand measured with X-ray absorption (crosses) and fitted van Genuchten parameterisation (solid line). The light grey vertical line indicates the left border of the range of data used for the fit 
A possible explanation for the deviation between the two sets of parameters is the uncertainty of the $\psi$-axis in the $\mathrm{X}$ ray absorption due to experimental difficulties. The determination of $\alpha$ is very sensitive to this. Also, in Fig. 2, a prediction using the same $\alpha$ as found in the best-fit of the outflow data is shown (bright grey). This indicates that $\alpha$ needs more careful determination.

The analysis of the multi-step outflow experiment allows parameter estimation, at least in principle, without reaching hydrostatic equilibrium; this is not the case for the simple analysis of the X-ray scanning experiment. An obvious extension is to combine the two approaches and use time series of X-ray scans during a multi-step outflow experiment. This will greatly enhance understanding of the processes and allow a much better determination of the effective parameters. Nevertheless, a rather quick alternative to estimating hydraulic properties has been demonstrated; this is particularly attractive for coarse-grained materials.

\section{DYNAMIC EXPERIMENTS}

In this experiment, the detector was mounted vertically to determine the distribution of water along the $z$-axis with a very high temporal resolution (Fig. 4). Initially, (during the first thirty minutes), water content changed very rapidly but it reached an almost stationary state after some 16 hours. More interestingly, however, Fig. 4 reveals that the dynamics of capillary rise cannot be described by the Richards' equation. Indeed with $\psi=0 \mathrm{~cm}$ at the lower boundary in direct contact with the water table - it would predict full saturation from the beginning of the experiment. However, the column was not saturated immediately at the bottom and, after a rapid increase initially, the water content increased rather slowly. The dynamics for this rise of water into the dry column may be described by two processes, an initial fast imbibition leading to a water content of $\theta=0.29$ after six minutes followed by a slow process that increases the water content at the bottom slowly to the stationary value of about 0.35 . This may result from the loss of continuity of the air phase during the imbibition. In the beginning, water enters the column rapidly and fills the pores with small diameters faster than the larger ones because of stronger capillary forces. Water may flow around some pores quickly and cut off the gas phase, leading to entrapment of air which will then slowly bubble through the overlying water barrier as pressure in the air phase increases. However, some fraction of the air may not be removed in this way and may remain in the column for a long time as a residual air phase. In the present experiment, this phase occupied a volume fraction of about 0.05 corresponding to a residual air saturation of about $12 \%$ of the pore space. This phase will

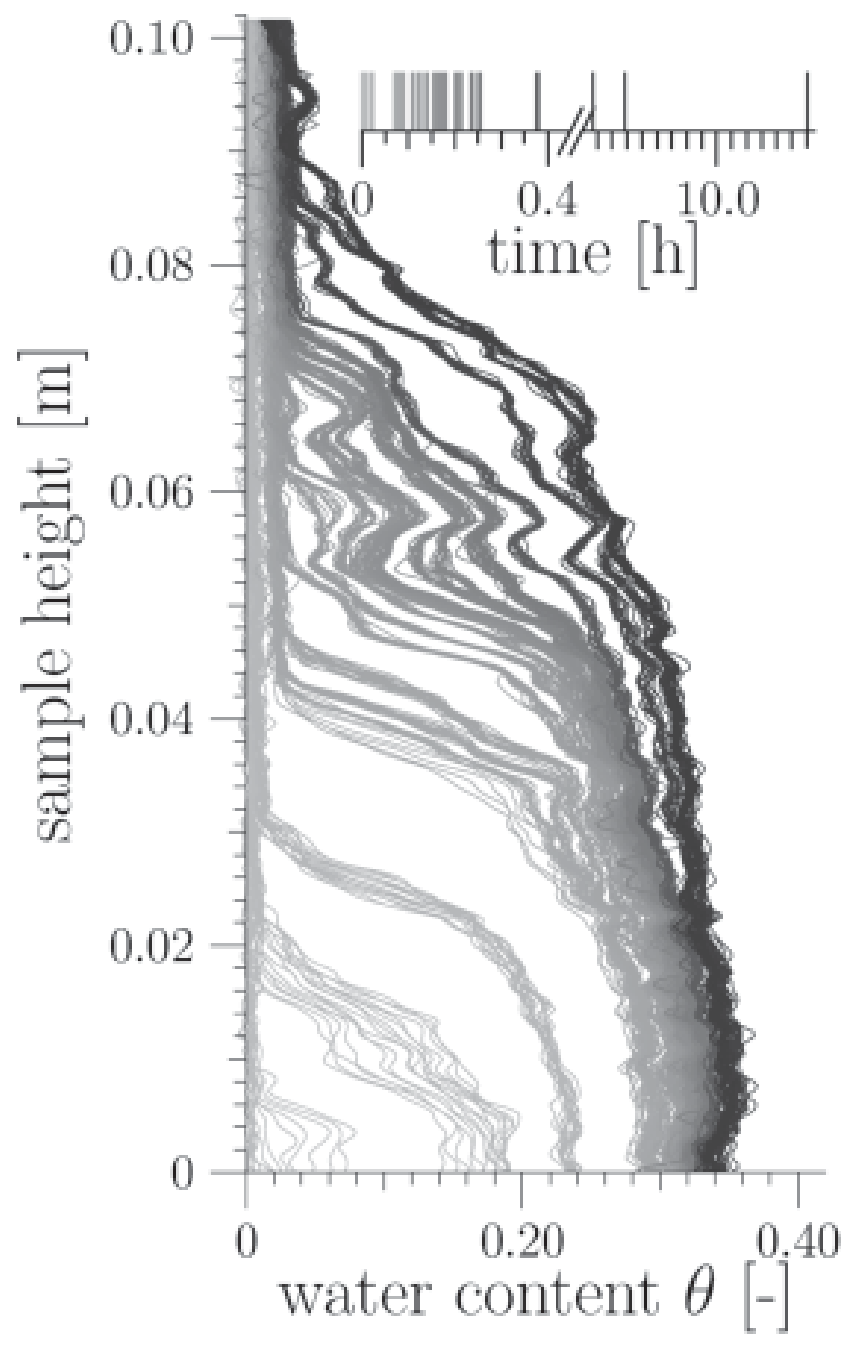

Fig. 4. Water content $v$. the sample height during the capillary rise of water in a dry porous column, the different times of imbibition are colour coded. Individual lines in a group are separated by $1 \mathrm{~s}$.

disappear only by exceedingly slow diffusion of air through the water phase.

\section{Conclusions}

Direct and non-invasive measurement of water content in rigid porous media opens new perspectives in determining the effective hydraulic properties of materials through modified outflow experiments. Such methods would be particularly useful for coarse-textured media which are otherwise very difficult to handle. In addition, it has been shown that, at least in the initial phase, capillary rise into a dry porous medium cannot be described by the Richards' equation, even if the medium is uniform. However, the observed phenomena can be understood qualitatively in terms of the formation of a residual air phase and of its 
partial disappearance with increasing pressure. While this phenomenon is interesting per se because it illuminates the essential multiphase nature of flow in the vadose zone, it also has important implications, for instance for the analysis of classical multi-step outflow experiments, or for air entrapment near the water table, the notorious 'excess air problem' with environmental tracers (Aeschbach-Hertig et al., 1999).

\section{References}

Aeschbach-Hertig, W., Peeters, F., Beyerle, U. and Kipfer, R., 1999. Interpretation of dissolved atmospheric noble gases in natural waters. Water Resour. Res., 35, 2779-2792.

Brown, J.M., Fonteno, W.C., Cassel, D.K. and Johnson, G.A., 1987. Computed tomographic analyses of water distribution in three porous foam media. Soil Sci. Soc. Amer. J., 51, 11211125.

Celia, M.A., Bouloutas, E.T. and Zarba, R.L., 1990. A general mass-conservative numerical solution for the unsaturated flow equation. Water Resour. Res., 26, 1483-1496.

Garnier, P., Angulo-Jaramillo, R., DiCarlo, D.A., Bauters, T.W. J., Darnault, C.J.G., Steenhuis, T.S., Parlange, J.-Y. and Baveye, P., 1998. Dual-energy synchrotron X-ray measurements of rapid soil density and water content changes in swelling soils during infiltration. Water Resour. Res., 34, 2837-2842.

Hopmans, J.W., Vogel, T. and Koblik, P.D., 1992. X-ray tomography of soil water distibution in one-step outflow experiments. Soil Sci. Soc. Amer. J., 56, 355-362.
Ketcham, A. and Carlson, D., 2001. Acquisition, optimization and interpretation of X-ray computed tomographic imagery: applications to the geosciences. Comput. Geosci., 27, 381400.

Klute, A., (Ed.) 1986. Methods of Soil Analysis. 1: Physical and Mineralogical Methods. Agronomy Series 9, 2nd edition, American Society of Agronomy, Madison, WI, USA.

Microcal, 1997. Microcal Origin, Users's Manual, Microcal Software, Inc., One Roundhouse Plaza, Northampton, MA 01060, USA.

Mori, Y., Maruyama, T. and Mitsuno, T., 1999. Soft X-ray radiography of drainage patterns of structured soils. Soil Sci. Soc. Amer. J., 63, 733-740.

Press, W.H., Teukolsky, S.A., Vetterling, W.T. and Flannery, B.P., 1992. Numerical recipes in C. Cambridge University Press, Cambridge, UK.

Rogasik, H., Crawford, J.W., Wendroth, O., Young, I.M. and Ritz, K., 1999. Discrimination of soil phases by dual energy X-ray tomography. Soil Sci. Soc. Amer. J., 63, 741-751.

van Dam, J.C., Stricker, J.N.M. and Droogers, P., 1994. Inverse method to determine soil hydraulic functions from multistep outflow experiments. Soil Sci. Soc. Amer. J., 58, 647-652.

van Genuchten, M.T., 1980. A closed-form equation for predicting the hydraulic conductivity of unsaturated soils. Soil Sci. Soc. Amer. J., 44, 892-898.

Wildenschild, D., Hopmans, J.W., Vaz, C.M.P., Rivers, M.L., Rikard, D. and Christensen, B.S.B., 2002. Using X-ray computed tomography in hydrology: systems, resolutions, and limitations. J. Hydrol., 267, 285-297.

Zurmuhl, T., 1994. Validierung konvektiv-dispersiver Modelle zur Berechnung des instatioären Stofftransports in ungestörten Bodensäulen, Bayreuther Bodenkundliche Berichte, Band 36. 